\title{
DETERMINAÇÃO TURBIDIMÉTRICA EM FLUXO DE CLORIDRATO DE FLUOXETINA EM FORMULAÇÕES FARMACÊUTICAS
}

\author{
Willian Toito Suarez, Elen Romão Sartori, Érica Ferreira Batista e Orlando Fatibello-Filho* \\ Departamento de Química, Universidade Federal de São Carlos, CP 676, 13560-970 São Carlos - SP, Brasil
}

Recebido em 29/1/09; aceito em 22/5/09; publicado na web em 10/11/09

\begin{abstract}
FLOW-INJECTION TURBIDIMETRIC DETERMINATION OF FLUOXETINE HYDROCHLORIDE IN PHARMACEUTICAL FORMULATIONS. A simple, accurate and precise flow-injection turbidimetric procedure for the determination of fluoxetine hydrochloride in pharmaceutical formulations is reported. The procedure is based on the precipitation of chloride of fluoxetine hydrochloride with silver nitrate solution and the yielded insoluble $\mathrm{AgCl}_{(\mathrm{s})}$ was monitored at $420 \mathrm{~nm}$. The analytical curve was linear in the fluoxetine hydrochloride concentration range $3.0 \times 10^{-5}-5.0 \times 10^{-4} \mathrm{~mol} \mathrm{~L}^{-1}$ with a detection limit of $10 \mu \mathrm{mol} \mathrm{L}^{-1}$ and, a sample throughout of $60 \mathrm{~h}^{-1}$.
\end{abstract}

Keywords: flow injection analysis; fluoxetine hydrochloride; turbidimetry.

\section{INTRODUÇÃO}

O cloridrato de fluoxetina, N-metil- $\gamma$-[4-(trifluorometil)fenoxi]benzenopropanamina, é uma droga antidepressiva, extremamente ativa como inibidor seletivo da recaptação da serotonina no sistema nervoso central, sendo amplamente prescrita no tratamento de várias condições médicas, dentre outras, depressão, transtorno obsessivo compulsivo, síndrome do pânico e fobia social. ${ }^{1}$

A fluoxetina atua corrigindo as concentrações inadequadas de serotonina no cérebro, que causa os sintomas na situação de doença. Sendo uma amina secundária, é desmetilada a norfluoxetina, composto também ativo. Ela não exibe efeitos anticolinérgicos e hipotensores como os antidepressivos tricíclicos (ADT), pois não bloqueia os receptores muscarínicos, serotonérgicos, dopaminérgicos, histaminérgicos e adrenérgicos. ${ }^{1,2}$

Devido à sua importância farmacológica e terapêutica, além de relativa ausência de reações adversas graves e baixo potencial de abuso, o cloridrato de fluoxetina tornou-se um dos antidepressivos mais utilizados no tratamento de alguns transtornos neurológicos. As reações adversas mais comuns relacionados à fluoxetina, mesmo em doses terapêuticas, são: boca seca, sudorese, cefaleia, diarreia, sonolência e insônia. Quando em doses excessivas pode ser nociva à saúde, levando até a morte do paciente. Dessa forma, é importante um controle do teor do medicamento administrado pelo mesmo, ${ }^{3}$ a fim de garantir o sucesso do tratamento e a minimização dos efeitos colaterais. Isso gera um interesse crescente no desenvolvimento de métodos analíticos simples e viáveis para a determinação deste analito em amostras comerciais.

As farmacopeias oficiais, como a britânica e a americana, recomendam o uso da cromatografia líquida de alta eficiência (CLAE) com detecção UV para a determinação do cloridrato de fluoxetina em medicamentos. ${ }^{4,5}$ Vários outros métodos têm sido desenvolvidos para a quantificação desse analito em formulações farmacêuticas como, por exemplo, cromatografia líquida ${ }^{6,7}$ e gasosa,${ }^{8}$ eletroforese capilar, ${ }^{9}$ fluorimetria, ${ }^{9,10}$ quimiluminescência, ${ }^{11}$ condutometria, ${ }^{12}$ voltametria ${ }^{13,14} \mathrm{e}$ espectrofotometria, ${ }^{6,15-21}$ alguns dos quais adaptados em sistemas por injeção em fluxo. Muitos desses métodos são sofisticados, morosos, empregam reagentes tóxicos, em muitos casos, seguidos de extração por solventes orgânicos, meios reacionais desfavoráveis, longo tempo

*e-mail: bello@ufscar.br reacional, além da necessidade de instrumentação de elevado custo para o emprego em laboratórios de análises de rotina.

O emprego da turbidimetria em sistema FIA tem sido possível, uma vez que a detecção em sistemas em fluxo é realizada com a amostra em movimento, evitando a sedimentação das partículas formadas em linha. Além disso, o emprego de surfactantes contribui para que o tamanho das partículas seja o mais uniforme possível, além de manter o precipitado em suspensão, produzindo sinais analíticos com uma melhor repetibilidade.

O emprego de medidas turbidimétricas em sistemas de análise por injeção em fluxo tem sido amplamente explorado no nosso grupo de trabalho, ${ }^{22-27}$ especialmente para a quantificação de diversos fármacos. ${ }^{24-27}$ Suarez et al. ${ }^{24}$ e Canaes e Fatibello-Filho ${ }^{25}$ empregaram sistema de análise em fluxo (FIA) com detecção turbidimétrica para a determinação de acetilcisteína e metilbrometo de homatropina, respectivamente. Ambos os procedimentos baseiam-se na precipitação do analito de interesse com nitrato de prata. Outros reagentes precipitantes têm sido empregados. Como exemplo, Costa-Neto et al. ${ }^{26}$ desenvolveram um método em fluxo (FIA) para a determinação de tiamina em amostras comerciais, empregando ácido silicotungstico. Marcolino-Jr. et al..$^{27}$ empregaram um reator em fase sólida contendo $\mathrm{AgCl}(\mathrm{s})$ acoplado a um sistema FIA de linha única para a determinação turbidimétrica de dipirona em formulações farmacêuticas.

No presente trabalho, descreve-se a determinação turbidimétrica do cloridrato de fluoxetina em formulações farmacêuticas, empregando-se análise por injeção em fluxo com zonas coalescentes e fluxo intermitente. Neste método, o cloreto do cloridrato de fluoxetina foi precipitado com nitrato de prata, sendo a suspensão de $\mathrm{AgCl}_{(\mathrm{s})}$ monitorada turbidimetricamente em $420 \mathrm{~nm}$.

\section{PARTE EXPERIMENTAL}

\section{Instrumentação}

As medidas turbidimétricas em fluxo foram feitas em um espectrofotômetro de feixe simples (Femto, Brasil), modelo 435, equipado com cela de fluxo construída em vidro com caminho óptico de 1,00 $\mathrm{cm}$ e volume de $180 \mu \mathrm{L}$, em $420 \mathrm{~nm}$, conectado a um registrador $\mathrm{x}-\mathrm{t}$ de dois canais Cole Parmer (Niles, IL, USA), modelo 1202.0000. Para propulsão das soluções de referência, reagentes e amostras utilizou-se uma bomba peristáltica de 12 canais (Ismatec IPC-12, 
Zurich, Suíça), modelo 7618-50 e tubos de propulsão de Tygon ${ }^{\circledR}$. Para a confecção das bobinas helicoidais, alça de amostragem e das linhas de condução das soluções de referência, amostras e reagentes foram empregados tubos e conexões de polietileno com diâmetros internos de $0,8 \mathrm{~mm}$. As amostras, soluções de referência e reagentes foram inseridas no sistema de fluxo com auxílio de um injetor comutador manual, construído em acrílico. ${ }^{28}$

\section{Reagentes e soluções}

Todos os reagentes empregados foram de grau analítico e todas as soluções foram preparadas com água destilada e desionizada (resistividade $\geq 18,2 \mathrm{M} \Omega \mathrm{cm}$ a $25^{\circ} \mathrm{C}$ ) obtida de um sistema de purificação Milli-Q (Millipore ${ }^{\circledR}$, Bedford, MA), modelo UV Plus ultrabaixo teor de substâncias orgânicas dissolvidas.

Cloridrato de fluoxetina (Lilly, Brasil), $\mathrm{AgNO}_{3}$ (Aldrich), $\mathrm{HNO}_{3}$ (Merck) e Triton X-100 (Merck) foram utilizados neste trabalho.

A solução estoque de cloridrato de fluoxetina $5,0 \times 10^{-3} \mathrm{~mol} \mathrm{~L}^{-1}$ foi preparada dissolvendo-se uma massa adequada deste sal em solução de Triton X-100 0,03\% (v/v) em meio de ácido nítrico 1,0 x 10-2 mol $\mathrm{L}^{-1}$. As demais soluções de cloridrato de fluoxetina foram preparadas por meio de diluições adequadas com solução de Triton X-100 0,03\% (v/v) em meio de ácido nítrico $1,0 \times 10^{-2} \mathrm{~mol} \mathrm{~L}^{-1}$.

A solução de nitrato de prata $1,0 \times 10^{-3} \mathrm{~mol} \mathrm{~L}^{-1}$ foi preparada dissolvendo-se uma massa adequada deste sal em água desionizada.

A solução estoque de Triton X-100 0,03\% (v/v) foi preparada diluindo-se uma alíquota adequada da solução concentrada de Triton $\mathrm{X}$-100 em meio de ácido nítrico $1,0 \times 10^{-2} \mathrm{~mol} \mathrm{~L}^{-1}$.

\section{Sistema de análise por injeção em fluxo}

O diagrama esquemático do sistema de análise por injeção em fluxo com zonas coalescentes empregado para a determinação turbidimétrica de cloridrato de fluoxetina em formulações farmacêuticas é mostrado na Figura 1. Nesse procedimento, as soluções de referências/amostras de cloridrato de fluoxetina e do reagente $\mathrm{AgNO}_{3} 1,0 \mathrm{x}$ $10^{-3} \mathrm{~mol} \mathrm{~L}^{-1}$ foram introduzidas simultaneamente com o auxílio do injetor-comutador na solução transportadora (Triton X-100 0,03\% (v/v) em meio de ácido nítrico $\left.1,0 \times 10^{-2} \mathrm{~mol} \mathrm{~L}^{-1} ; 2,4 \mathrm{~mL} \mathrm{~min}^{-1}\right)$ por meio de alças de $350 \mu \mathrm{L}$. Os volumes injetados da amostra e do reagente percorrem distâncias iguais até se confluírem no ponto $\mathrm{X}$, onde ocorre a formação da suspensão de $\mathrm{AgCl}_{(\mathrm{s})}$ (reação do cloreto do cloridrato de fluoxetina com $\mathrm{Ag}(\mathrm{I})$ do reagente), sendo a seguir monitorada turbidimetricamente em $420 \mathrm{~nm}$. O sinal transiente obtido foi proporcional à concentração de cloridrato de fluoxetina injetado no sistema.

Quando o injetor comutador está na posição de amostragem (como mostrado na Figura 1), um fluxo intermitente de água desionizada, a uma vazão de $6,3 \mathrm{~mL} \mathrm{~min}^{-1}$, entra no ponto de confluência Y, efetuando a limpeza da bobina helicoidal B e da cela de fluxo de caminho óptico de $1,0 \mathrm{~cm}$.

\section{Preparação das formulações farmacêuticas e determinação do cloridrato de fluoxetina}

Foram realizadas determinações de cloridrato de fluoxetina em cinco produtos comerciais disponíveis no mercado nacional, sendo três amostras na forma de cápsulas e duas líquidas, denominadas A, B, C, D e E.

Na preparação das amostras líquidas, $1,0 \mathrm{~mL}$ de cada amostra contendo cloridrato de fluoxetina foi precisamente transferida para balões volumétricos de $50,0 \mathrm{~mL}$, completou-se os volumes com solução de Triton X-100 0,03\% (v/v) em meio de ácido nítrico 1,0 x

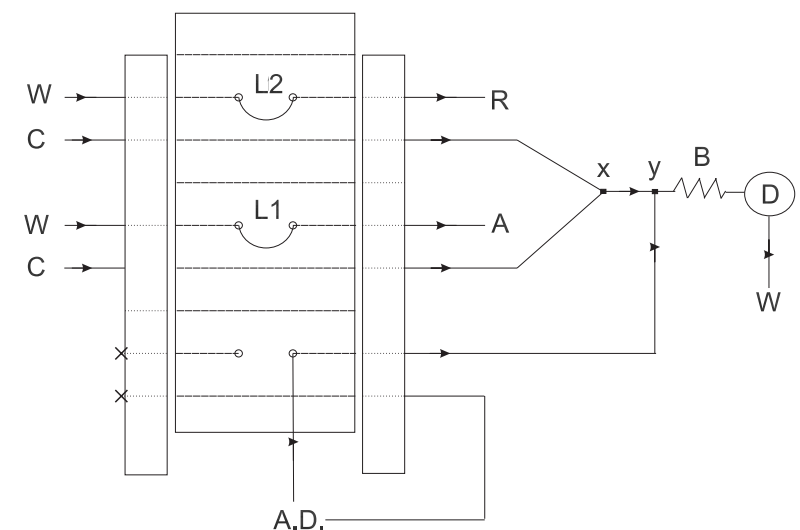

Figura 1. Diagrama esquemático do sistema de análise por injeção em fluxo com zonas coalescentes e fluxo intermitente para determinação turbidimétrica de cloridrato de fluoxetina em formulações farmacêuticas. As representações são: $I=$ injetor comutador; $A$ = amostra ou solução de referência; $R=$ reagente $\mathrm{AgNO}_{3}\left(1,0 \times 10^{-3} \mathrm{~mol} \mathrm{~L}^{-1}\right) ; \mathrm{L1}=$ alça de amostragem $(350 \mu \mathrm{L}) ; \mathrm{L2}=$ alça de reagente $(350 \mu \mathrm{L}) ; C=$ solução transportadora $($ Triton $X-1000,03 \%$ em meio de ácido nítrico $\left.1,0 \times 10^{-2} \mathrm{~mol} \mathrm{~L}^{-1} ; 2,4 \mathrm{~mL} \mathrm{~min}^{-1}\right) ; B=$ bobina helicoidal (100 cm); $X$ = ponto de confluência; $Y=$ entrada do fluxo intermitente de água

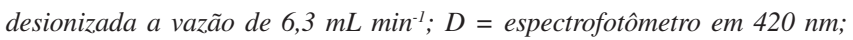
$W=$ descarte e $A D=$ água desionizada $\left(6,3 \mathrm{~mL} \mathrm{~min}^{-1}\right)$

$10^{-2} \mathrm{~mol} \mathrm{~L}^{-1}$. Em seguida, transferiu-se um volume adequado dessas soluções para balões volumétricos de $25,0 \mathrm{~mL}$, completando-se o volume com solução Triton X-100 0,03\% (v/v) em meio de ácido nítrico $1,0 \times 10^{-2} \mathrm{~mol} \mathrm{~L}^{-1}$.

Para a preparação das amostras sólidas, dez cápsulas de cada amostra foram pesadas em balança analítica e pulverizadas em almofariz. Uma quantidade adequada foi dissolvida em balão volumétrico de $100 \mathrm{~mL}$ com solução de Triton X-100 0,03\% (v/v) em meio de ácido nítrico $1,0 \times 10^{-2} \mathrm{~mol} \mathrm{~L}^{-1}$. Os excipientes insolúveis contidos nas amostras foram removidos por filtração simples em papel de filtro Whatman $n^{\circ} 1$. Uma alíquota adequada do filtrado foi transferida para balões volumétricos de $50,0 \mathrm{~mL}$, completando-se o volume com solução de Triton X-100 0,03\% (v/v) em meio de ácido nítrico $1,0 \times 10^{-2} \mathrm{~mol} \mathrm{~L}^{-1}$.

\section{RESULTADOS E DISCUSSÃO}

\section{Estudos preliminares}

O sistema de análise por injeção em fluxo com zonas coalescentes e fluxo intermitente com detecção turbidimétrica proposto neste trabalho para a determinação de cloridrato de fluoxetina em produtos farmacêuticos baseia-se na reação entre o cloreto do cloridrato de fluoxetina e o nitrato de prata $\left(\mathrm{AgNO}_{3}\right)$, formando o precipitado de $\mathrm{AgCl}_{(\mathrm{s})}$, que foi monitorado turbidimetricamente em $420 \mathrm{~nm}$. À medida que os íons cloreto reagem com os íons $\mathrm{Ag}_{(\text {(aq) }}$, os íons $\mathrm{H}^{+}$ $\left(\mathrm{H}_{3} \mathrm{O}^{+}\right)$devem continuar na molécula (protonação do amino grupo da fluoxetina), como mostrado na Figura 2.

Inicialmente, foram estudados os parâmetros químicos do sistema em fluxo para investigar as melhores condições para a formação do $\mathrm{AgCl}_{(\mathrm{s})}$ em linha.

Tipicamente, a formação de precipitado em linha tende a sedimentar na bobina helicoidal e na cela de fluxo, provocando a elevação da linha base. Para eliminar esse acúmulo de precipitado, foi inserido na configuração inicial do sistema um fluxo intermitente com água desionizada, a uma vazão de $6,3 \mathrm{~mL} \mathrm{~min}^{-1}$, como fluxo de limpeza. Essa configuração permitiu um aumento na frequência de amostragem de 30 para $60 \mathrm{~h}^{-1}$ e uma melhor estabilidade da linha base. 

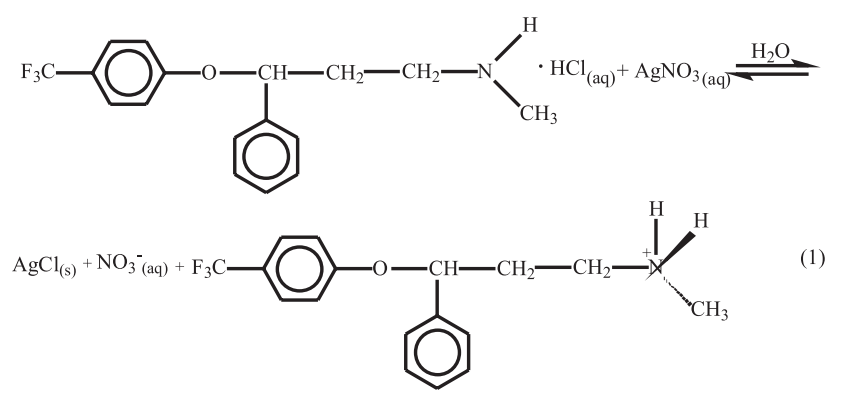

Figura 2. Reação de formação do $\mathrm{AgCl}_{(s)}$ a partir do $\mathrm{AgNO}_{3(a q)}$ e o cloridrato de fluoxetina

O estudo para a otimização da melhor solução transportadora foi realizado em um sistema FIA com volume das alças da amostra e do reagente de $350 \mu \mathrm{L}$ cada, vazão da solução transportadora de $1,7 \mathrm{~mL}$ $\min ^{-1}$ e bobina helicoidal de $120 \mathrm{~cm}$. A concentração de cloridrato de fluoxetina foi mantida em $1,0 \times 10^{-3} \mathrm{~mol} \mathrm{~L}^{-1}$.

Ácido nítrico na faixa de concentração de $1,0 \times 10^{-3}$ a 5,0 x 10-2 mol $\mathrm{L}^{-1}$ e água desionizada foram avaliadas como soluções transportadoras. Os melhores resultados em termos de magnitude do sinal analítico foram obtidos empregando-se água desionizada. Por outro lado, os melhores resultados em termos de repetibilidade dos sinais analíticos foram obtidos utilizando-se ácido nítrico $1,0 \times 10^{-2} \mathrm{~mol} \mathrm{~L}^{-1}$. Dessa forma, selecionou-se o ácido nítrico $1,0 \times 10^{-2} \mathrm{~mol} \mathrm{~L}^{-1}$ como solução transportadora, além de que este ajuda a manter a força iônica do meio constante e evitar a hidrólise do $\mathrm{Ag}(\mathrm{I})$.

A adição de colóides protetores ou surfactantes nas soluções é muitas vezes requerida, pois a presença destes agentes é uma garantia adicional de nucleação uniforme do precipitado, melhorando a repetibilidade e a magnitude dos sinais analíticos. Dessa forma, estudou-se o efeito do polietilenoglicol (PEG), álcool polivinílico (PVA), Triton X-100 e Agar-Agar nas concentrações de 0,010, 0,030 e 0,050\% (m/v) sobre o sinal analítico, estabilidade da linha base e repetibilidade dos sinais transientes. Os melhores resultados em termos de repetibilidade e magnitude dos sinais analíticos foram obtidos utilizando-se Triton X-100 0,030\% (v/v). Em virtude desses resultados, selecionou-se o surfactante Triton X-100 na concentração de $0,030 \%$ v/v para o preparo da solução transportadora e da amostra.

Estudou-se a influência da concentração do reagente nitrato de prata no intervalo de concentração de 5,0 x10-4 a 5,0 x $10^{-2} \mathrm{~mol} \mathrm{~L}^{-1}$ sobre o sinal analítico, nas condições experimentais apresentadas na Figura 1. Uma maior magnitude de sinal analítico e melhor estabilidade da linha base foram obtidas para a concentração de $1,0 \mathrm{x}$ $10^{-3} \mathrm{~mol} \mathrm{~L}^{-1}$ desse reagente de precipitação, sendo selecionada essa concentração. Para concentrações maiores, houve uma diminuição pouco acentuada do sinal analítico (tendência à estabilização) e perda da estabilidade da linha base.

O efeito da variação dos volumes da amostra e do reagente foi estudado variando-se simultaneamente as alças L1 (amostra) e L2 (reagente). Os volumes das alças foram variados no intervalo de 200 a $500 \mu \mathrm{L}$ (comprimentos de 40, 50, 70 e $100 \mathrm{~cm}$ ), sendo mantida a concentração de cloridrato de fluoxetina em $1,0 \times 10^{-3} \mathrm{~mol} \mathrm{~L}^{-1}$. Neste estudo, observou-se que a magnitude do sinal analítico aumentou até um volume de amostra e reagente injetado de $500 \mu \mathrm{L}$. Houve um aumento progressivo do sinal analítico, devido a menor dispersão da zona da amostra com o aumento do volume injetado. Porém, devido a uma melhor repetibilidade dos sinais analíticos e uma maior frequência de amostragem, o volume de $350 \mu \mathrm{L}$ foi selecionado para continuidade do trabalho.

A influência do comprimento da bobina helicoidal (colocada após o ponto de confluência X, Figura 1) sobre o sinal analítico foi avaliada no intervalo de 70 a $120 \mathrm{~cm}$. A magnitude do sinal analítico aumenta até o comprimento de $100 \mathrm{~cm}$, diminuindo para o comprimento da bobina de $120 \mathrm{~cm}$, provavelmente devido ao efeito de dispersão. Como a cinética da reação de formação do precipitado é rápida, a dispersão prevalece e a turbidez diminui. Considerando-se a magnitude do sinal analítico, a bobina helicoidal de $100 \mathrm{~cm}$ foi selecionada para dar sequência aos estudos.

$\mathrm{O}$ efeito da vazão da solução transportadora (ácido nítrico $1,0 \mathrm{x}$ $10^{-2} \mathrm{~mol} \mathrm{~L}^{-1}$ em Triton X-100 0,03\% (v/v)) sobre o sinal analítico foi estudada no intervalo entre 2,2 e $4,1 \mathrm{~mL} \mathrm{~min}^{-1}$. Utilizando-se uma solução de cloridrato de fluoxetina na concentração de $1,0 \times 10^{-3}$ mol $\mathrm{L}^{-1}$, o sinal analítico diminuiu com o aumento da vazão, possivelmente, devido a um menor tempo de residência para a formação do precipitado. Observou-se uma maior magnitude de sinal analítico e melhor estabilidade da linha base empregando-se a menor vazão $\left(2,2 \mathrm{~mL} \mathrm{~min}^{-1}\right)$, que foi então selecionada para estudos posteriores.

A Tabela 1 apresenta os parâmetros avaliados e os parâmetros selecionados na otimização do sistema em fluxo para a determinação de cloridrato de fluoxetina em formulações farmacêuticas.

Tabela 1. Parâmetros avaliados na otimização do sistema em fluxo para a determinação de cloridrato de fluoxetina empregando o sistema FIA proposto

\begin{tabular}{|c|c|c|}
\hline Parâmetros & Faixa estudada & Selecionado \\
\hline$\left[\mathrm{AgNO}_{3}\right] / 10^{-2} \mathrm{~mol} \mathrm{~L}^{-1}$ & 0,05 a 5 & 0,1 \\
\hline $\begin{array}{l}\text { alça da amostra e do } \\
\text { reagente/ } \mu \mathrm{L}\end{array}$ & 100 a 500 & 350 \\
\hline bobina helicoidal $/ \mathrm{cm}$ & 70 a 120 & 100 \\
\hline $\begin{array}{l}\text { vazão da solução trans- } \\
\text { portadora } / \mathrm{mL} \min ^{-1}\end{array}$ & 2,2 a 4,1 & 2,2 \\
\hline surfactantes & $\begin{array}{l}\text { PVA }^{\#} \text {, PEG*, Triton } \\
\text { X-100 e Agar-Agar }\end{array}$ & Triton X-100 \\
\hline
\end{tabular}

\#álcool polivinílico; *polietilenoglicol.

\section{Curva analítica}

A curva analítica obtida apresenta linearidade no intervalo de concentração de cloridrato de fluoxetina entre $3,0 \times 10^{-5}$ e 5,0 x 10 $10^{-4}$ mol L ${ }^{-1}$ e pode ser descrita pela equação $\mathrm{T}=0,00372+387,004$ [cloridrato de fluoxetina], $\mathrm{r}=0,998$ onde $\mathrm{T}$ é a turbidez e [cloridrato de fluoxetina] a concentração de cloridrato de fluoxetina em $\mathrm{mol} \mathrm{L}^{-1}$, com limite de detecção (três vezes o desvio padrão do branco/inclinação da curva analítica) de $1,0 \times 10^{-5} \mathrm{~mol} \mathrm{~L}^{-1}$.

A repetibilidade do procedimento desenvolvido foi determinada para concentrações de soluções de referência de cloridrato de fluoxetina de $2,0 \times 10^{-4}$ e $3,0 \times 10^{-4} \mathrm{~mol} \mathrm{~L}^{-1}$, obtendo-se desvios padrões relativos de 0,5 e $2,9 \%$, respectivamente. A frequência de amostragem foi de $60 \mathrm{~h}^{-1}$.

\section{Estudo de interferentes em potencial no método proposto}

Foram avaliados alguns excipientes normalmente presentes em formulações farmacêuticas que podem interferir na determinação de cloridrato de fluoxetina. As substâncias avaliadas foram sacarina sódica, metabissulfito de sódio, estearato de magnésio, celulose microcristalina, dióxido de silício, amido e ciclamato de sódio, em várias razões de concentração, sendo o cloridrato de fluoxetina mantido na concentração de $3,0 \times 10^{-4} \mathrm{~mol} \mathrm{~L}^{-1}$ para este estudo. Alguns destes excipientes são insolúveis e/ou possuem baixa solubilidade. Estearato de magnésio, ciclamato de sódio e amido não causaram interferência na determinação 
de cloridrato de fluoxetina, mesmo quando presentes em concentrações dez vezes maiores que aquela de fluoxetina. Entretanto, a sacarina sódica apresentou interferência positiva de $7 \%$ quando presente em concentração dez vezes maior que aquela do cloridrato de fluoxetina. A interferência positiva da sacarina se deve à formação do sacarinato de prata, de baixa solubilidade, aumentando assim a turbidez da zona de amostra e, consequentemente, a magnitude do sinal analítico. O metabissulfito apresentou interferência negativa de $15 \%$ quando presente na mesma concentração do cloridrato de fluoxetina. Essa interferência ocorre devido ao poder de redução desse concomitante reduzindo a $\mathrm{Ag}^{+} \mathrm{a} \mathrm{Ag}^{\circ}$, que promove uma diminuição na formação do precipitado de $\mathrm{AgCl}$. Cabe ressaltar que os teores desses excipientes nos produtos farmacêuticos são inferiores aos investigados nesse estudo.

\section{Teste de recuperação}

No teste de recuperação, três concentrações diferentes de cloridrato de fluoxetina, a saber: $1,0 \times 10^{-5}, 4,0 \times 10^{-5}$ e $6,0 \times 10^{-5} \mathrm{~mol}$ $\mathrm{L}^{-1}$, foram adicionadas a cinco amostras comerciais e os resultados comparados com aqueles obtidos com as amostras não adicionadas do padrão. Os valores percentuais variaram entre 96,0 e $103 \%$ sugerindo, assim, ausência de interferência significativa da matriz da amostra no procedimento proposto.

\section{Aplicação analítica}

O método proposto foi aplicado na determinação de cloridrato de fluoxetina em cinco formulações farmacêuticas A, B, C, D e E, tratadas conforme descrito na parte experimental e os resultados obtidos foram comparados com o método cromatográfico descrito na Farmacopéia Americana. ${ }^{5}$

A Tabela 2 apresenta os resultados obtidos para essas determinações. A concentração de cloridrato de fluoxetina em cada amostra foi determinada diretamente na curva analítica.

Tabela 2. Determinação de cloridrato de fluoxetina em formulações farmacêuticas empregando-se o sistema FIA proposto e o método oficial ${ }^{5}$

\begin{tabular}{|c|c|c|c|c|}
\hline \multirow{2}{*}{ Amostras } & \multicolumn{3}{|c|}{ Cloridrato de fluoxetina } & \multirow{2}{*}{$\begin{array}{c}\text { Erro } \\
\text { Relative } \\
/ \%\end{array}$} \\
\hline & Rotulado & Método Oficial $^{\mathrm{a}}$ & Proposto $^{a}$ & \\
\hline$(\mathrm{A})^{*}$ & 20,00 & $19,60 \pm 0,01$ & $19,20 \pm 0,03$ & $-4,1$ \\
\hline$(\mathrm{B})^{*}$ & 22,36 & $22,20 \pm 0,02$ & $23,30 \pm 0,01$ & 4,0 \\
\hline$(C)^{* * *}$ & 22,36 & $22,00 \pm 0,01$ & $23,00 \pm 0,02$ & 2,8 \\
\hline$(\mathrm{D})^{* *}$ & 22,36 & $22,40 \pm 0,03$ & $22,32 \pm 0,01$ & $-0,2$ \\
\hline$(E)^{* * *}$ & 22,40 & $21,90 \pm 0,02$ & $21,40 \pm 0,03$ & $-4,7$ \\
\hline
\end{tabular}

Valores em:* $\mathrm{mg} \mathrm{mL}^{-1} \mathrm{e}^{* *} \mathrm{mg}$ cápsula ${ }^{-1} ;{ }^{\mathrm{a}} \mathrm{n}=5$; Erro Relativo = proposto vs. método oficial.

O teste-t pareado ${ }^{29}$ foi aplicado aos resultados obtidos empregando ambos os métodos, resultando em um valor de $\mathrm{t}$ calculado de 0,545 , menor que o valor de t tabelado $(2,776)$, indicando que o procedimento proposto possui boa exatidão, uma vez que não foi observada diferença significativa entre os resultados obtidos, a um nível de confiança de $95 \%$.
As características analíticas do método proposto foram comparadas com aquelas obtidas por outros procedimentos em fluxo para a determinação de cloridrato de fluoxetina, como mostrado na Tabela 3. Como pode ser observado nesta tabela, o método proposto possui uma linearidade menor que aquelas obtidas pelos demais métodos, um limite de detecção menor que aquele obtido no método espectrofotométrico ${ }^{19}$ e superior àqueles obtidos nos demais métodos. ${ }^{11,21}$ Outrossim, a frequência de amostragem foi igual àquela obtida no método espectrofotométrico ${ }^{21} \mathrm{e}$ foi menor que aquelas obtidas nos demais métodos. ${ }^{11,19}$

Tabela 3. Características analíticas dos procedimentos em fluxo para a determinação de cloridrato de fluoxetina

\begin{tabular}{lcccc}
\hline Detecção & $\begin{array}{c}\text { Linearidade / } \\
\mathrm{mol} \mathrm{L}^{-1}\end{array}$ & $\begin{array}{c}\mathrm{LD} / \\
\mu \mathrm{mol} \mathrm{L}{ }^{-1}\end{array}$ & $\begin{array}{c}\text { Frequência de } \\
\text { amostragem / } \\
\mathrm{h}^{-1}\end{array}$ & Ref. \\
\hline $\begin{array}{l}\text { Quimilumi- } \\
\text { nescente }\end{array}$ & $0-2,9 \times 10^{-5}$ & 0,89 & 136 & 11 \\
$\begin{array}{l}\text { Espectrofoto- } \\
\text { métrica }\end{array}$ & $\begin{array}{c}5,0 \times 10^{-5}- \\
1,0 \times 10^{-2}\end{array}$ & 25 & 80 & 19 \\
$\begin{array}{l}\text { Espectrofoto- } \\
\text { métrica }\end{array}$ & $\begin{array}{c}1,4 \times 10^{-6}- \\
7,2 \times 10^{-5}\end{array}$ & 0,43 & 60 & 21 \\
Turbidimétrica & $\begin{array}{c}3,0 \times 10^{-5}- \\
5,0 \times 10^{-4}\end{array}$ & 10 & 60 & Proposto \\
\hline
\end{tabular}

LD = Limite de Detecção

\section{CONCLUSÕES}

O método proposto para a determinação do cloridrato de fluoxetina em diferentes formulações farmacêuticas, tanto em cápsulas como em amostras líquidas, mostra-se como uma alternativa econômica e rápida em análise rotineira de controle de qualidade desse fármaco na indústria farmacêutica e em farmácias de manipulação. Ademais, o método é simples, preciso e exato, com reduzido consumo de amostra/reagente quando comparado com os procedimentos espectrofotométricos em fluxo descritos na literatura. ${ }^{19,21}$ Além disso, as amostras podem ser analisadas sem a necessidade de tratamento especial ou do emprego de algum tipo de solvente orgânico, ${ }^{21}$ sem produção de resíduos que causariam impacto ambiental.

\section{AGRADECIMENTOS}

Ao CNPq, à CAPES e FAPESP pelo apoio financeiro e à Lilly do Brasil pelo cloridrato de fluoxetina cedido, bem como ao CNPq pela bolsa de doutorado de W. T. Suarez, à CAPES pela bolsa de doutorado de E. R. Sartori e à FAPESP pela bolsa de iniciação científica de E. F. Batista (Proc. nº 2007/07692-4).

\section{REFERÊNCIAS}

1. Oliveira, I. R. Em Farmacologia; Silva, P., ed.; Guanabara Koogan S.A.: Rio de Janeiro, 2000, cap. 34.

2. Baldessarini, R. J. Em Goldman \& Gilman - As Bases Farmacológicas da Terapêutica; Brunton, L. L.; Lazo, J. S.; Parker, K. L., eds.; Mc Graw-Hill: Rio de Janeiro, 2006, cap. 17.

3. http://www.who.int/factsheet/fs275/en, acessada em Janeiro 2009.

4. British Pharmacopoeia, Stationary Office: London, 2000.

5. The United States Pharmacopoeia, $24^{\text {th }}$ ed., United States Pharmacopoeial Convenction: Rockville, 2000.

6. Raggi, M.A; Bugamelli, F.; Casamenti, G.; Mandrioli, R.; De Ronchi, D.; Volterra, V.; J. Pharm. Biomed. Anal. 1998, 18, 699. 
7. El-Dawy, M. A.; Mabrouk, M. M.; El-Barbary, F. A.; J. Pharm. Anal. 2002, 30, 561 .

8. Berzas Nevado, J. J.; Villaseor Llerena, M. J.; Contento Salcedo, A. M.; Aguas Nuevo, E.; J. Pharm. Biomed. Anal. 2005, 38, 52.

9. Mandrioli, R.; Pucci, V.; Visini, D.; Varani, G.; Raggi, M. A.; J. Pharm. Biomed. Anal. 2002, 29, 1127.

10. Berzas, J. J.; Alañón, A.; Lázaro, J. A.; Talanta 2002, 58, 301.

11. Marques, K. L.; Santos, J. L. M.; Lima, J. L. F. C.; Anal. Lett. 2007, 40, 2241.

12. Sartori, E. R.; Suarez, W. T.; Fatibello-Filho, O.; Anal. Lett. 2009, 42, 659.

13. Roque da Silva, A. M. S.; Lima, J. C.; Oliva Teles, M. T.; Oliveira Brett, A. M.; Talanta 1999, 49, 611 .

14. Nows, H. P. A.; Delerue-Matos, C.; Barros, A. A.; Rodrigues, J. A.; Santos-Silva, A.; Borges, F.; Anal. Lett. 2007, 40, 1131.

15. Prabhakar, A. H.; Patel, V. B.; Giridhar, R.; J. Pharm. Biomed. Anal. 1999, 20, 427.

16. Bebawy, L. I.; El-Kousy, N.; Suddik, J. K.; Shokry, M.; J. Pharm. Biomed. Anal. 1999, 21, 133.

17. Starczewska, B.; Mielich, K.; J. Pharm. Biomed. Anal. 2000, 23, 243.

18. Starczewska, B.; Puzanowska-Tarasiewicz, H.; Baranowska, K.; J. Pharm. Biomed. Anal. 2000, 23, 477.
19. Afkhami, A.; Madrakian, T.; Khalafi, L.; Chem. Pharm. Bull. 2006, 54, 1642.

20. Fregonezi-Neri, M. M.; Baracat, M. M.; Casagrande, R.; Machado, H. T.; Miglioranza, B.; Gianotto, E. A. S.; Dalmas, J. C.; Quim. Nova 2008, 31,1665

21. Shah, J.; Jan, M. R.; Rehman, F.; J. Chilean Chem. Soc. 2008, 53, 1605.

22. Diniz, M. C. T.; Oliveira, A. F.; Fatibello-Filho, O.; Lab. Rob. Autom. 2000, 12, 236.

23. Piccin, E.; Vieira, H. J.; Fatibello-Filho, O.; Anal. Lett. 2005, 38, 511.

24. Suarez, W. T.; Vieira, H. J.; Fatibello-Filho, O.; J. Braz. Chem. Soc. 2007, 18, 1028 .

25. Canaes, L. S.; Fatibello-Filho, O.; Quim. Nova 2006, 29, 1237.

26. Costa-Neto, C. O.; Pereira, A. V.; Aniceto, C.; Fatibello-Filho, O.; Talanta 1999, 48, 659.

27. Marcolino-Jr., L. H.; Bonifácio, V. G.; Fatibello-Filho, O.; Teixeira, M. F. S.; Quim. Nova 2005, 28, 783.

28. Bergamin, F. H.; Reis, B. F.; Zagatto, E. A. G.; Anal. Chim. Acta 1978, 97, 427.

29. Anderson, R. L.; Practical Statistics for Analytical Chemists, D. Van Nostrand Reinhold Company: New York, 1987. 